\title{
A Synchronous Optimization of Magnitude and Placement of Multiple Clamping Forces for Fixture Design
}

\author{
Tiejun $\mathrm{Wu}^{1}$ and Guohua Qin ${ }^{2, *}$ \\ ${ }^{1}$ Department of Mechanical and Electronics Engineering, Dongguan Polytechnic, \\ Dongguan, China \\ ${ }^{2}$ School of Aeronautical Manufacturing Engineering, Nanchang Hangkong University, \\ Nanchang, China \\ ${ }^{1}$ wtj1980@126.com, ${ }^{2, *}$ qghwzx@126.com
}

\begin{abstract}
In order to resist external loads, multiple clamping forces will be produced to a workpiece so that its correct position can be immobilized during the entire machining operation. However, insufficient clamping forces cannot prevent the workpiece from motions whereas excessive clamping forces may cause the improper deformations of workpiece-fixture system. Therefore, a novel approach to clamping force determination is proposed for fixture design. Firstly, the measure method of magnitudes and placements of clamping forces is established from the various measure spaces to the uniform measure space for the first time. Secondly, a synchronous optimal model of magnitudes and placements of clamping forces is formulated as nonlinear constrained equations according to the minimum total complementary energy principle. By solving the resulting nonlinear equations, all contact forces including clamping forces and their application placements can accurately predicted in the frictional workpiecefixture system. This method is illustrated with example cases. The method presented here may also have an application to other passive, indeterminate problems such as power grasps in robotics.
\end{abstract}

Keywords: Multiple clamping forces, Workpiece position error, Synchronous optimization, Total complementary energy

\section{Introduction}

Fixtures are used to locate and immobilize workpieces with locator(s) and clamp(s) so that the desired positions and orientations of a workpiece can be maintained during manufacturing processes, such as machining, assembly, inspection, welding, and so on. The locating correctness is a primary requirement in fixture design, and it is related to the locators' number and their layout in the fixture $[1,2]$. Another crucial requirement is workpiece stability which would ensure that when a suitable set of clamping forces applied on the workpiece, the workpiece subjected to the external can keep in contact with the fixture elements with finite workpiece position error. Therefore, this paper focuses on modeling and determining of magnitudes and placements of clamping forces.

The determination and optimization of clamping forces has been widely studied. Under the assumption of rigid workpiece-fixture system, Chou et al. applied the screw theory to formulate clamping force determination as a linear constrained optimal problem [3]. With the consideration of the elastic contact deformations between the workpiece and fixture elements, $\mathrm{Li}$ and Melkote utilized the minimum total complementary principle to optimize the clamping 
forces where as Gui et al. evaluated the clamping forces by minimizing the overall contact deflections [4, 5]. Marin and Ferreira presented an algebraic method based on screw theory and the solution of systems of linear inequalities to compute the dependent application regions of clamping forces on a workpiece with a predefined 3-2-1 locator scheme [6]. Xiong et al. used a nonlinear programming method to derive the minimum number of frictionless contact points needed and the way to arrange them to achieve form-closure [7]. However, these researches separate the magnitudes and placements of clamping forces into two independent design problems.

Assumed design variations to be independent with each other, Chen et al. employed the finite element method to determine magnitudes and placements of clamping forces as a constrained optimization model and simultaneously developed a genetic algorithm to solve the optimization model [8]. The stable region of fixturing position, however, must be known in advance. Moreover, a common limitation of this approach is the large model size and computation cost.

An efficient method is devised for synchronous determination of magnitudes and placements of the clamping forces. A unit force method is firstly proposed to determine the fixel stiffness. And then, a kinematic model is used to determine a set of contact forces including clamping forces based on the uniform measure criterion of magnitudes and placements of clamping forces. The method seeks to minimize the effect of workpiece motion due to clamping and machining loads on the workpiece position error by optimizing the clamping forces and displacements.

\section{Fixel Stiffness}

As shown in Figure $1, \boldsymbol{n}_{i}=\left[n_{i x}, n_{i y}, n_{i z}\right]^{\mathrm{T}}, \boldsymbol{t}_{i}=\left[t_{i x}, t_{i y}, t_{i z}\right]^{\mathrm{T}}$ and $\boldsymbol{\tau}_{i}=\left[\tau_{i x}, \tau_{i y}, \tau_{i z}\right]^{\mathrm{T}}$ are the unit inner normal vector and two orthogonal unit tangential vectors of the workpiece at contact point $\boldsymbol{r}_{i}=\left[x_{i}, y_{i}, z_{i}\right]^{\mathrm{T}}$ of the $i$ th fixel, respectively. Contacts between the workpiece and fixels are considered as frictional contacts. For the $i$ th fixel, $\boldsymbol{F}_{i}=\boldsymbol{F}_{i n}+\boldsymbol{F}_{i t}+\boldsymbol{F}_{i \tau}$ is the $i$ th resultant contact force with $\boldsymbol{F}_{i n}, \boldsymbol{F}_{i t}$ and $\boldsymbol{F}_{i \tau}$ being the three components of $\boldsymbol{F}_{i}$ along $\boldsymbol{n}_{i}, \boldsymbol{t}_{i}$ and $\boldsymbol{\tau}_{i}$, respectively. Hence,

$$
\boldsymbol{F}_{i n}=f_{i n} \boldsymbol{n}_{i}, \boldsymbol{F}_{i t}=f_{i t} \boldsymbol{t}_{i}, \boldsymbol{F}_{i \tau}=f_{i \tau} \boldsymbol{\tau}_{i}
$$

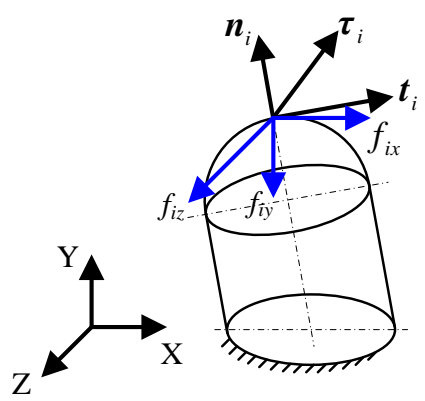

\section{Figure 1. The Forced Status of a Fixel}

Denote $k_{i n}, k_{i t}$ and $k_{i \tau}$ three components of fixel stiffness at the $\boldsymbol{n}_{i}, \boldsymbol{t}_{i}$ and $\boldsymbol{\tau}_{i}$ directions. Assumed that the fixel is subject to the unit force $\boldsymbol{F}_{i}^{*}=\left[F_{i x}^{*}, F_{i y}^{*}, F_{i z}^{*}\right]^{\mathrm{T}}$ with $F_{i x}^{*}=1, F_{i y}^{*}=1$ and $F_{i z}^{*}=1$ in the $\mathrm{X}, \mathrm{Y}$ and $\mathrm{Z}$ directions. Thus, the unit deformation $\boldsymbol{U}_{i}^{*}$ and the unit stiffness $\boldsymbol{K}_{i}^{*}$ of the $i$ th fixel in $\{\mathrm{X}-\mathrm{Y}-\mathrm{Z}\}$ can be easily computed by finite element 
method. Therefore, the fixel deformation can be mapped from $\{\mathrm{X}-\mathrm{Y}-\mathrm{Z}\}$ to $\left\{\boldsymbol{n}_{i}-\boldsymbol{t}_{i}-\boldsymbol{\tau}_{i}\right\}$ following

$$
\boldsymbol{u}_{i}^{*}=\boldsymbol{T}\left(\boldsymbol{\Theta}_{i}\right)^{\mathrm{T}} \boldsymbol{U}_{i}^{*}
$$

where $\boldsymbol{T}\left(\Theta_{i}\right)=\left[\boldsymbol{n}_{i}, \boldsymbol{t}_{i}, \boldsymbol{\tau}_{i}\right]$ is the transform matrix of $i$ th contact point.

Likewise, the unit force can be transformed from $\{\mathrm{X}-\mathrm{Y}-\mathrm{Z}\}$ to $\left\{\boldsymbol{n}_{i}-\boldsymbol{t}_{i}-\boldsymbol{\tau}_{\boldsymbol{i}}\right\}$ as

$$
\boldsymbol{f}_{i}^{*}=\boldsymbol{T}\left(\boldsymbol{\Theta}_{i}\right)^{\mathrm{T}} \boldsymbol{F}_{i}^{*}
$$

with $\boldsymbol{f}_{i}^{*}=\left[f_{i n}^{*}, f_{i t}^{*}, f_{i \tau}^{*}\right]^{\mathrm{T}}$ being described in $\left\{\boldsymbol{n}_{i}-\boldsymbol{t}_{i}-\boldsymbol{\tau}_{i}\right\}$.

Therefore, the relationship between the force $\boldsymbol{f}_{i}^{*}$ and the deformation $\boldsymbol{u}_{i}$ can be obtained as

$$
\boldsymbol{f}_{i}^{*}=\boldsymbol{k}_{i}^{*} \boldsymbol{u}_{i}^{*}
$$

where $\boldsymbol{k}_{i}=\operatorname{diag}\left(k_{i n}, k_{i t}, k_{i \tau}\right)$ is the fixel stiffness matrix.

By substituting Eqs. $(2,3)$ into Eq. (4), the fixel stiffness can be achieved as

$$
\boldsymbol{k}_{i}^{*}=\boldsymbol{T}\left(\boldsymbol{\Theta}_{i}\right)^{\mathrm{T}} \boldsymbol{K}_{i}^{*} \boldsymbol{T}\left(\boldsymbol{\Theta}_{i}\right)
$$

Now, the practical contact force at $i$ th fixel is assumed to be $f_{i}=\left[f_{i n}, f_{i t}, f_{i \tau}\right]^{\mathrm{T}}$. Consequently, it can be transformed from $\left\{\boldsymbol{n}_{i}-\boldsymbol{t}_{i}-\boldsymbol{\tau}_{i}\right\}$ to $\{\mathrm{X}-\mathrm{Y}-\mathrm{Z}\}$ as

$$
\boldsymbol{F}_{i}=\boldsymbol{T}\left(\boldsymbol{\Theta}_{i}\right) \boldsymbol{f}_{i}
$$

Since the relationship between the practical contact force and the unit contact force can be written as

$$
\boldsymbol{F}_{i}=\operatorname{diag}\left(\boldsymbol{F}_{i}\right) \boldsymbol{F}_{i}^{*}=\operatorname{diag}\left(\boldsymbol{F}_{i}\right) \boldsymbol{K}_{i}^{*} \boldsymbol{U}_{i}^{*}
$$

The combination of Eq. (6) with Eq. (7) gives rise to

$$
\boldsymbol{f}_{i}=\boldsymbol{T}\left(\boldsymbol{\Theta}_{i}\right)^{\mathrm{T}} \operatorname{diag}\left(\boldsymbol{T}\left(\boldsymbol{\Theta}_{i}\right) \boldsymbol{f}_{i}\right) \boldsymbol{K}_{i}^{*} \boldsymbol{T}\left(\boldsymbol{\Theta}_{i}\right) \boldsymbol{u}_{i}^{*}
$$

Hence, the fixel stiffness can be derived from Eq. (8) as

$$
\boldsymbol{k}_{i}=\boldsymbol{T}\left(\boldsymbol{\Theta}_{i}\right)^{\mathrm{T}} \operatorname{diag}\left(\boldsymbol{T}\left(\boldsymbol{\Theta}_{i}\right) \boldsymbol{f}_{i}\right) \boldsymbol{K}_{i}^{*} \boldsymbol{T}\left(\boldsymbol{\Theta}_{i}\right)
$$

\section{Workpiece Position Error}

Based on the hook law, the following equation can be obtained

$$
\boldsymbol{f}_{i}=\boldsymbol{k}_{i} \boldsymbol{\delta}_{i}
$$

where $\boldsymbol{\delta}_{i}$ is the local deformation at $i$ th contact joint.

As shown in Figure 2, the local deformation $\boldsymbol{\delta}_{i}$ can cause the workpiece position variation. Here, $\boldsymbol{r}_{\mathrm{w}}, \boldsymbol{\Theta}_{\mathrm{w}}$ and $\boldsymbol{r}_{\mathrm{f} i}, \boldsymbol{\Theta}_{\mathrm{f} i}$ are the position and orientation of the workpiece and the $i$ th fixel with respect with $\{\mathrm{X}-\mathrm{Y}-\mathrm{Z}\}$, respectively. Thus,

$$
\boldsymbol{r}_{\mathrm{w}}+\boldsymbol{T}\left(\boldsymbol{\Theta}_{\mathrm{w}}\right) \boldsymbol{r}_{i}^{\mathrm{w}}=\boldsymbol{r}_{\mathrm{f} i}+\boldsymbol{T}\left(\boldsymbol{\Theta}_{\mathrm{f} i}\right) \boldsymbol{r}_{i}^{\mathrm{f}}, 1 \leq i \leq m+n
$$

where $\boldsymbol{T}\left(\boldsymbol{\Theta}_{\mathrm{w}}\right)$ is the transform matrix from $\left\{\mathrm{X}^{\mathrm{w}}-\mathrm{Y}^{\mathrm{w}}-\mathrm{Z}^{\mathrm{w}}\right\}$ to $\{\mathrm{X}-\mathrm{Y}-\mathrm{Z}\}$ whereas $\boldsymbol{T}\left(\boldsymbol{\Theta}_{\mathrm{f} i}\right)$ is the transform matrix from $\left\{\mathrm{X}^{\mathrm{f}}-\mathrm{Y}^{\mathrm{f}}-\mathrm{Z}^{\mathrm{f}}\right\}$ to $\{\mathrm{X}-\mathrm{Y}-\mathrm{Z}\}$. 
Eq. (11) is differentiated with respect to $\boldsymbol{r}_{\mathrm{w}}, \boldsymbol{\Theta}_{\mathrm{w}}, \boldsymbol{r}_{i}^{\mathrm{w}}$ and $\boldsymbol{r}_{i}^{\mathrm{f}}$ such that

$$
\delta \boldsymbol{r}_{\mathrm{w}}+\delta \boldsymbol{T}\left(\boldsymbol{\Theta}_{\mathrm{w}}\right) \boldsymbol{r}_{i}^{\mathrm{w}}=\boldsymbol{\delta}_{i}, 1 \leq i \leq m+n
$$

where $\boldsymbol{\delta}_{i}=\boldsymbol{T}\left(\Theta_{i}\right) \boldsymbol{\kappa}_{1} f_{i}$ and $\boldsymbol{k}_{i}=\operatorname{diag}\left(\boldsymbol{k}_{i n}, \boldsymbol{k}_{i t}, \boldsymbol{k}_{i \tau}\right)$ are the local deformation and local stiffness matrix.

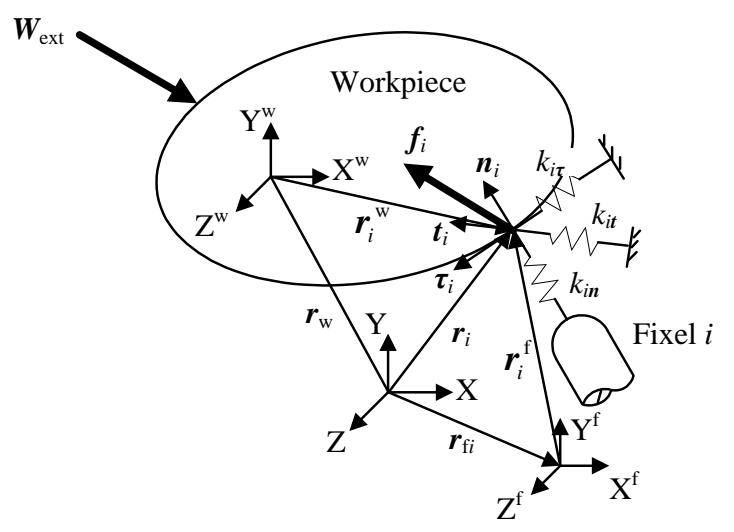

Figure 2. A Workpiece-Fixture System

Under the assumption of the identification of $\left\{\mathrm{X}^{\mathrm{w}}-\mathrm{Y}^{\mathrm{w}}-\mathrm{Z}^{\mathrm{w}}\right\}$ and $\left\{\mathrm{X}^{\mathrm{f}}-\mathrm{Y}^{\mathrm{f}}-\mathrm{Z}^{\mathrm{f}}\right\}$ with $\{\mathrm{X}-$ Y-Z $\}$ in all directions, $\boldsymbol{T}\left(\boldsymbol{\Theta}_{\mathrm{w}}\right)$ and $\delta \boldsymbol{T}\left(\boldsymbol{\Theta}_{\mathrm{w}}\right)$ will be the unit and skew symmetric matrices. Then the workpiece position error can be expressed as

$$
\boldsymbol{e} \Delta \boldsymbol{q}_{\mathrm{w}}=\boldsymbol{T}(\boldsymbol{\Theta}) \boldsymbol{k}^{-1} \boldsymbol{f}
$$

where $\Delta \boldsymbol{q}_{\mathrm{w}}=\left[\delta \boldsymbol{r}_{\mathrm{w}}^{\mathrm{T}}, \delta \Theta_{\mathrm{w}}^{\mathrm{T}}\right]^{\mathrm{T}}$ is the workpiece position error. $\boldsymbol{T}(\Theta)=\operatorname{diag}\left[\boldsymbol{T}\left(\Theta_{1}\right), \boldsymbol{T}\left(\boldsymbol{\Theta}_{2}\right), \ldots\right.$, $\left.\boldsymbol{T}\left(\Theta_{m+n}\right)\right]$ is the transform matrix, $\boldsymbol{e}=\left[\boldsymbol{e}_{1}^{\mathrm{T}}, \boldsymbol{e}_{2}^{\mathrm{T}}, \ldots, \boldsymbol{e}_{m+n}^{\mathrm{T}}\right]^{\mathrm{T}}$ with $\boldsymbol{e}_{i}=\left[\boldsymbol{e}_{x}, \boldsymbol{e}_{y}, \boldsymbol{e}_{z}, \boldsymbol{r}_{i} \times \boldsymbol{e}_{x}, \boldsymbol{r}_{i} \times \boldsymbol{e}_{y}\right.$, $\left.\boldsymbol{r}_{i} \times \boldsymbol{e}_{z}\right], \boldsymbol{e}_{x}=[1,0,0]^{\mathrm{T}}, \boldsymbol{e}_{y}=[0,1,0]^{\mathrm{T}}$ and $\boldsymbol{e}_{z}=[0,0,1]^{\mathrm{T}}$ is the configuration matrix, $\boldsymbol{k}=\operatorname{diag}\left(\boldsymbol{k}_{1}, \boldsymbol{k}_{2}\right.$, $\left.\ldots, \boldsymbol{k}_{m+n}\right)$ is the fixel stiffness matrix, and $\boldsymbol{f}=\left[\boldsymbol{f}_{1}^{\mathrm{T}}, \boldsymbol{f}_{2}^{\mathrm{T}}, \ldots, \boldsymbol{f}_{m+n}^{\mathrm{T}}\right]^{\mathrm{T}}$ is the contact force vector including clamping forces.

It is worthy to notice that the workpiece position error can be further represented as follows if the workpiece is assumed to be correct location and equilibrium status [9].

$$
\Delta \boldsymbol{q}_{\mathrm{w}}=\boldsymbol{e}^{+} \boldsymbol{T}(\boldsymbol{\Theta}) \boldsymbol{k}^{-1} \boldsymbol{f}
$$

where $\boldsymbol{e}^{+}$is a Moore-Penrose inverse matrix of $\boldsymbol{e}$.

\section{Optimal Model}

Insufficient clamping forces cannot prevent the workpiece from motions whereas excessive clamping forces may cause highly the improper deformations of workpiecefixture system. In order to resolve the conflict, clamping forces must be optimized.

\subsection{Static Equilibrium Constraints}

As shown in Figure 2, a workpiece-fixture system consists of one workpiece, $m$ locators and $n$ clamps. The workpiece-fixture system must be in static equilibrium for a stable fixture configuration so that 


$$
\boldsymbol{G} \boldsymbol{f}+\boldsymbol{W}_{\mathrm{ext}}=\boldsymbol{O}
$$

where $\boldsymbol{G}=\left[\boldsymbol{G}_{1}, \boldsymbol{G}_{2}, \ldots, \boldsymbol{G}_{m+n}\right]$ is the orientation matrices with $\boldsymbol{G}_{i}=\left[\boldsymbol{G}_{i \boldsymbol{n}}, \boldsymbol{G}_{\boldsymbol{i}}, \boldsymbol{G}_{\boldsymbol{i \tau}}\right], \boldsymbol{G}_{\boldsymbol{i n}}=[\boldsymbol{n}$ $\left.{ }_{i}^{\mathrm{T}},\left(\boldsymbol{r}_{i} \times \boldsymbol{n}_{i}\right)^{\mathrm{T}}\right]^{\mathrm{T}}, \boldsymbol{G}_{i t}=\left[\boldsymbol{t}_{i}^{\mathrm{T}},\left(\boldsymbol{r}_{i} \times \boldsymbol{t}_{i}\right)^{\mathrm{T}}\right]^{\mathrm{T}}$ and $\boldsymbol{G}_{i \boldsymbol{\tau}}=\left[\boldsymbol{\tau}_{i}^{\mathrm{T}},\left(\boldsymbol{r}_{i} \times \boldsymbol{\tau}_{i}\right)^{\mathrm{T}}\right]^{\mathrm{T}}, \boldsymbol{f}=\left[\boldsymbol{f}_{1}^{\mathrm{T}}, \boldsymbol{f}_{2}^{\mathrm{T}}, \ldots, \boldsymbol{f}_{m+n}^{\boldsymbol{T}}\right]^{\mathrm{T}}$ is the contact force including clamping force, and $\boldsymbol{W}_{\text {ext }}$ is the external wrench.

\subsection{Friction Cone Constraints}

It is theoretically known that the resultant of the normal and frictional forces at any contact point must also lie within the friction cone to prevent the workpiece from slipping. Assumed that $\mu_{i}$ is the friction coefficient between the workpiece and the $i$ th fixel, the following inequation can be obtained according to Coulomb's Friction Law

$$
\operatorname{diag}\left(\boldsymbol{f}^{\mathrm{T}}\right) \boldsymbol{\mu} \operatorname{diag}(\boldsymbol{f}) \leq \boldsymbol{0}
$$

where $\boldsymbol{\mu}_{=} \operatorname{diag}\left(\boldsymbol{\mu}_{1}, \boldsymbol{\mu}_{2}, \ldots, \boldsymbol{\mu}_{m+n}\right)$ is the friction coefficient matrix with $\boldsymbol{\mu}_{i}=\operatorname{daig}\left(-\mu_{i}, 1,1\right)$.

\subsection{Direction Constraints}

In order to prevent the workpiece from detaching from the fixels in the fixturing process, the normal forces at any contact point between the workpiece and the fixel must be in compression such that

$$
\boldsymbol{n}^{\mathrm{T}} \boldsymbol{T}(\boldsymbol{\Theta}) \boldsymbol{f} \geq \mathbf{0}
$$

where $\boldsymbol{n}=\operatorname{diag}\left(\boldsymbol{n}_{1}, \boldsymbol{n}_{2}, \ldots, \boldsymbol{n}_{m+n}\right)$ is the normal vector matrix.

\subsection{Performance Constraint}

The normal compressive stress at the $i$ th contact point cannot exceed the compressive yield strength $\sigma_{\mathrm{s} i}$ of the workpiece material. This upper bound is written as

$$
\boldsymbol{n}^{\mathrm{T}} \boldsymbol{T}(\boldsymbol{\Theta}) \boldsymbol{f} \leq \boldsymbol{\sigma} \boldsymbol{A}
$$

where $\sigma=\operatorname{diag}\left(\sigma_{1}, \sigma_{2}, \ldots, \sigma_{m+n}\right)$ is the yield strength matrix, and $\boldsymbol{A}=\left[A_{1}, A_{2}, \ldots, A_{m+n}\right]^{\mathrm{T}}$ is the area vector with $A_{i}$ is the contact area at the $i$ th workpiece-fixture contact.

\subsection{Geometric Constraints}

A workpiece can only occupy the finite space. What's more, all of workpiece faces can not arrange the clamping forces. Let rlower and rupper be the lower bound and upper bound of clamping position, thus

$$
\boldsymbol{r}_{\text {lower }} \leq \boldsymbol{r} \leq \boldsymbol{r}_{\text {upper }}
$$

\subsection{Objective Function}

Contact forces are uniquely determined by means of the principle of minimum total complementary energy. The actual state of contact forces including clamping forces leads to an extreme value for the total complementary energy $\Pi$ with 


$$
\min \Pi=\frac{1}{2} \boldsymbol{f}^{\mathrm{T}} \boldsymbol{k}^{-1} \boldsymbol{f}
$$

Consequently, the solution approach to the synchronously optimization of clamping forces can be described as

$$
\begin{aligned}
& \text { find } \boldsymbol{r} \text { and } \boldsymbol{f} \\
& \min \Pi \\
& \text { s.t. } \Delta \boldsymbol{q}_{\mathrm{w}}^{\mathrm{T}} \Delta \boldsymbol{q}_{\mathrm{w}} \leq \varepsilon \\
& \\
& \boldsymbol{G} \boldsymbol{f}+\boldsymbol{W}_{\mathrm{ext}}=\boldsymbol{0} \\
& \operatorname{diag}\left(\boldsymbol{f}^{\mathrm{T}}\right) \boldsymbol{\mu} \operatorname{diag}(\boldsymbol{f}) \leq \boldsymbol{0} \\
& \boldsymbol{r}_{\text {lower }} \leq \boldsymbol{r} \leq \boldsymbol{r}_{\text {upper }} \\
& \boldsymbol{0} \leq \boldsymbol{n}^{\mathrm{T}} \boldsymbol{T}(\boldsymbol{\Theta}) \boldsymbol{f} \leq \boldsymbol{\sigma} \boldsymbol{A}
\end{aligned}
$$

where $\varepsilon$ is positive arbitrary number which is used to control the workpiece position error.

\section{Solution Technology}

In principle, variables $\boldsymbol{f}$ and $\boldsymbol{r}$ in Eq. (21) belong to force space and length space, respectively. Thus, the effects of $\boldsymbol{f}$ and $\boldsymbol{r}$ on convergence velocities of the objective function are unequal with each others. To circumvent this difficulty, the constraints in Eq. (12) must be performed the proper transformation to identify the space of $\boldsymbol{f}$ with $\boldsymbol{r}$. If $a$ and $b$ denote the components with the maximum absolute value in vectors $\sigma \boldsymbol{A}$ and $\boldsymbol{r}_{\text {upper }}$, several new notations are introduced for the static equilibrium constraints,

$$
a=\max \left(\boldsymbol{r}_{\text {upper }} \mid\right)
$$

and

$$
b=\max (|\boldsymbol{\sigma} \boldsymbol{A}|)
$$

If two members of Eqs. $(17,18)$ are simultaneously divided by $b$, then Eqs. $(17,18)$ can be transformed as

$$
\boldsymbol{0} \leq \boldsymbol{n}^{\mathrm{T}} \boldsymbol{T}(\boldsymbol{\Theta}) \boldsymbol{x}_{1} \leq \boldsymbol{B}
$$

where $\boldsymbol{x}_{1}=\boldsymbol{f} / b$, and $\boldsymbol{B}=\sigma \boldsymbol{A} / b$.

Likewise, the divide of Eq. (19) by $a$ can result in

$$
\boldsymbol{C} \leq \boldsymbol{x}_{2} \leq \boldsymbol{D}
$$

where $\boldsymbol{x}_{2}=\boldsymbol{r} / a, \boldsymbol{C}=\boldsymbol{r}_{\text {lower }} / a$, and $\boldsymbol{D}=\boldsymbol{r}_{\text {upper }} / a$.

In addition, Eq. (16) can be equivalently converted into

$$
\operatorname{diag}\left(\boldsymbol{x}_{1}^{\mathrm{T}}\right) \boldsymbol{\mu} \operatorname{diag}\left(\boldsymbol{x}_{1}\right) \leq \boldsymbol{0}
$$


Consequently, the solution approach to the synchronous optimization of $\boldsymbol{f}$ and $\boldsymbol{r}$ can be described as

$$
\begin{array}{ll}
\text { find } \boldsymbol{x}=\left[\boldsymbol{x}_{1}^{\mathrm{T}}, \boldsymbol{x}_{2}^{\mathrm{T}}\right]^{\mathrm{T}} \\
\min \Pi \\
\text { s.t. } & \Delta \boldsymbol{q}_{\mathrm{w}}^{\mathrm{T}} \Delta \boldsymbol{q}_{\mathrm{w}} \leq \boldsymbol{\varepsilon} \\
& \boldsymbol{G} \boldsymbol{f}+\boldsymbol{W}_{\text {ext }}=\boldsymbol{0} \\
& \operatorname{diag}\left(\boldsymbol{x}_{1}^{\mathrm{T}}\right) \boldsymbol{\mu} \operatorname{diag}\left(\boldsymbol{x}_{1}\right) \leq \boldsymbol{0} \\
& \boldsymbol{C} \leq \boldsymbol{x}_{2} \leq \boldsymbol{D} \\
& \boldsymbol{0} \leq \boldsymbol{n}^{\mathrm{T}} \boldsymbol{T}(\boldsymbol{\Theta}) \boldsymbol{x}_{1} \leq \boldsymbol{B}
\end{array}
$$

where $\varepsilon$ is positive arbitrary number, and $\boldsymbol{f}=b \boldsymbol{x}_{1}$.

\section{Numerical Tests}

To illustrate the approach, an example is shown in Figure 3. A through slot is milled from the top surface of a block workpiece with outline of $0.1 \mathrm{~m} \times 0.1 \mathrm{~m} \times 0.1 \mathrm{~m}$. Six locators A, B, C, D, E, F and three clamps AA, BB, CC have been setup to restrain the workpiece, whose coordinates and normal and tangential unit vectors are shown in Table 1. In the middle of the slot, the workpiece is subject to an external machining force $[0,358 \mathrm{~N},-497 \mathrm{~N}]^{\mathrm{T}}$ and machining torque $[0,0,9.47 \mathrm{Nm}]^{\mathrm{T}}$. The weight of the workpiece is $78 \mathrm{~N}$. Friction coefficient is $\mu=0.4$. According to Eq. (9), the fixel stiffness can be concluded as $k_{i n}=k_{i t}=k_{i \tau}=4.0 \times 10^{8} \mathrm{~N} / \mathrm{m}$. The compressive yield strength and the cross section area are $\sigma_{i}=160 \times 10^{6} \mathrm{~N} / \mathrm{m}^{2}$ and $A_{i}=254 \times 10^{-6} \mathrm{~m}^{2}$, respectively.

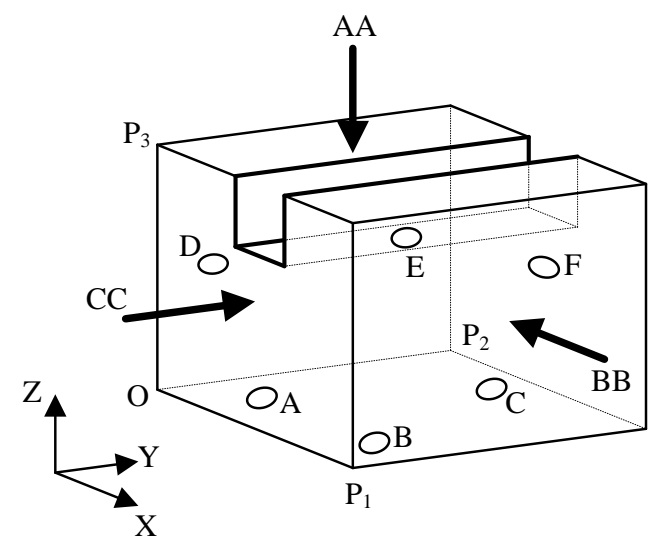

Figure 3. A Fixture with Six Locators and Three Clamps 
Table 1. Position and Orientation of Fixture Elements

\begin{tabular}{|c|c|c|c|c|}
\hline Fixels & $\begin{array}{c}\text { Coordinates } \\
\boldsymbol{r}_{i}(\mathrm{~m})\end{array}$ & $\begin{array}{c}\text { Normal } \\
\text { unit vectors } \boldsymbol{n}_{i}\end{array}$ & $\begin{array}{c}\text { Tangential } \\
\text { unit vectors } \boldsymbol{t}_{i}\end{array}$ & $\begin{array}{c}\text { Tangential } \\
\text { unit vectors } \boldsymbol{\tau}_{i}\end{array}$ \\
\hline A & $\begin{array}{c}(1.03,1.03,1 . \\
00)\end{array}$ & $(0,0,1)$ & $(1,0,0)$ & $(0,1,0)$ \\
\hline B & $\begin{array}{c}(1.07,1.03,1 . \\
00)\end{array}$ & $(0,0,1)$ & $(1,0,0)$ & $(0,1,0)$ \\
\hline C & $(1.07,1.03,1.00)$ & $(0,0,1)$ & $(1,0,0)$ & $(0,1,0)$ \\
\hline D & $\begin{array}{c}(1.05,1.07,1 . \\
00)\end{array}$ & $(1,0,0)$ & $(0,1,0)$ & $(0,0,1)$ \\
\hline E & $\begin{array}{c}(1.00,1.07,1 . \\
05)\end{array}$ & $(1,0,0)$ & $(0,1,0)$ & $(0,0,1)$ \\
\hline F & $\begin{array}{c}(1.00,1.03,1 . \\
05)\end{array}$ & $(0,-1,0)$ & $(1,0,0)$ & $(0,0,1)$ \\
\hline AA & --- & $(0,0,-1)$ & $(1,0,0)$ & $(0,-1,0)$ \\
\hline BB & --- & $(-1,0,0)$ & $(0,1,0)$ & $(0,0,-1)$ \\
\hline CC & --- & $(0,1,0)$ & $(0,0,1)$ & $(1,0,0)$ \\
\hline
\end{tabular}

The workable regions of clamping points $\mathrm{AA}, \mathrm{BB}$ and $\mathrm{CC}$ are listed respectively as Table 2.

Table 2. The Workable Regions of Clamping Points

\begin{tabular}{|c|c|c|c|}
\hline $\begin{array}{c}\text { Coordinate } \\
\mathrm{s}\end{array}$ & AA $(\mathrm{m})$ & $\mathrm{BB}(\mathrm{m})$ & $\mathrm{CC}(\mathrm{m})$ \\
\hline$X$ & {$[1.01,1.09]$} & 1.10 & {$[1.01,1.09]$} \\
\hline$Y$ & {$[1.01,1.09]$} & {$[1.01,1.09]$} & 1.10 \\
\hline$Z$ & 1.10 & {$[1.01,1.09]$} & {$[1.01,1.09]$} \\
\hline
\end{tabular}

By using MATLAB function fmincon to compute the clamping forces according to Eq. (27), the optimal placements of clamping forces are $x_{\mathrm{AA}}=1.09 \mathrm{~m}, y_{\mathrm{AA}}=1.01 \mathrm{~m}$, $z_{\mathrm{AA}}=1.10 \mathrm{~m}, \quad x_{\mathrm{BB}}=1.10 \mathrm{~m}, \quad y_{\mathrm{BB}}=1.0477 \mathrm{~m}, \quad z_{\mathrm{BB}}=1.0252 \mathrm{~m}, \quad x_{\mathrm{CC}}=1.01 \mathrm{~m}, \quad y_{\mathrm{CC}}=1.10 \mathrm{~m}$, $z_{\mathrm{CC}}=1.01 \mathrm{~m}$, whereas the optimal magnitudes of clamping forces are $f_{n \mathrm{AA}}=4.9668 \mathrm{~N}$, $f_{n \mathrm{BB}}=169.9112 \mathrm{~N}$, and $f_{n \mathrm{CC}}=28.9001 \mathrm{~N}$, as listed in Table 3 . In these conditions, the workpiece position error $\Delta \boldsymbol{q}_{\mathrm{w}}$ is obtained from Eq. (14) as $\delta \boldsymbol{r}_{\mathrm{w}}=[-0.659 \mu \mathrm{m}, 4.373 \mu \mathrm{m}$, $4.058 \mu \mathrm{m}]^{\mathrm{T}}$ and $\delta \Theta_{\mathrm{w}}=[0.6438 ",-0.1681 ",-0.2327 "]^{\mathrm{T}}$.

Table 3. Optimal Magnitudes and Placements

\begin{tabular}{|l|c|l|}
\hline Clamps & Placements $(\mathrm{m})$ & Magnitudes $(\mathrm{N})$ \\
\hline AA & $(1.09,1.01,1.10)$ & $(4.9668,-0.3397,-1.5947)$ \\
\hline BB & $(1.10,1.0477$, & $(169.9112,-37.6822,-35.3320)$ \\
\hline CC & $(1.0252)$ & \\
\hline
\end{tabular}




\section{Conclusions}

The clamping planning problem is one of the fundamental issues in fixture design. This paper focuses on the determination of the optimal magnitudes and positions of clamping forces. The optimization goal is to minimize the total complementary energy of the workpiece-fixture system. The problem is formulated as a constrained nonlinear programming. In order to carry out the synchronous optimization of magnitudes and positions of clamping forces, the uniform measure criterion of magnitudes and placements of clamping forces is sophisticatedly established. Moreover, the unit force method is proposed to obtain the fixel stiffness. The main advantage of the presented approach can be fit for complex workpiece with unknown stable clamping region.

\section{Acknowledgements}

This work is supported by Natural Science Foundation of China (51165039) and Scientific Research Foundation of Dongguan Polytechinic (2012a07).

\section{References}

[1] H. Song and Y. Rong, "Robotics and Computer-Integrated Manufacturing, vol. 21, (2005).

[2] G. H. Qin, W. H. Zhang and M. Wan, Transactions of the ASME Journal of Manufacturing Science and Engineering, vol. 130, (2008).

[3] Y. C. Chou, V. Chandru and M. M. Barash, Transactions of the ASME Journal of Engineering for Industry, vol. 111, (1989).

[4] B. Li and S. N. Melkote, International Journal of Advanced Manufacturing Technology, vol. 17, (2001).

[5] W. Gui, J. Y. H. Fuh and A. Y. C. Nee, IIE Transactions, vol. 28, (1996).

[6] R. A. Marin and P. M. Ferreira, Transactions of the ASME Journal of Manufacturing Science and Engineering, vol. 124, (2002).

[7] C. H. Xiong, Y. F. Li, Y. L. Xiong, H. Ding and Q. S. Huang, Robotics and Autonomous Systems, vol. 27, (1999).

[8] W. F. Chen, L. Ni and J. B. Xue, International Journal of Advanced Manufacturing Technology, vol. 38, (2008).

[9] G. H. Qin, W. H. Zhang and M. Wang, International Journal of Advanced Manufacturing Technology, vol. 29, (2006).

\section{Authors}

\section{Tiejun Wu}

He received the B.S. and M.S. degree from Central South University of Forestry, in 2001 and 2005 respectively. Now he is working for Dongguan Polytechnic. At the same time, he is studying for the doctor degree at Nanjing Aeronautics and Astronautics University. His research interest is computer-aided fixture design.

\section{Guohua Qin}

He received the M.S. and M.S. degree from Northwestern Polytechnical University, in 2003 and 2006 respectively. Now he is working for Nanchang Hangkong University. His research interest in computer-aided fixture design and the simulation modeling of machining process. 
International Journal of Control Automation Vol. 7, No. 6, (2014) 